\title{
Integration of maintenance systems
}

\author{
$S H$ Sarje \\ Department of Mechanical Engineering, JSPM's Imperial College of Engineering and Research, \\ Pune,India.
}

\begin{abstract}
Excellence in maintenance is imperative in highly competitive market because it resulted into minimum maintenance cost, high equipment effectiveness, maximum reliability of the system, high quality of the products, low delivery time, high flexibility, safety etc. Any maintenance system such as Total Productive Maintenance (TPM) or Reliability Centered Maintenance (RCM) or Condition Based Maintenance (CBM) alone cannot achieve the excellence in maintenance but its integration may do. In this paper, an integration of TPM, RCM and CBM is proposed with a maintenance policy to take advantage of their respective strengths. A continuously monitored system subject to degradation due to the imperfect maintenance, where a hybrid hazard rate based on the concept of age reduction factor and hazard rate increase factor to predict the evolution of the system reliability in different maintenance cycles has been assumed.A quantitative decision making model for an integrated maintenance system is derived in order to assess the performance of the proposed maintenance policy. Numerical examples of calculation of optimal preventive maintenance age $x$ and preventive maintenance number $\mathrm{N}^{*}$ for the given cost ratio of corrective replacement and predictive preventive maintenance are given.
\end{abstract}

\section{Introduction}

Cost effective maintenance along with quality of products, flexibility, maximum reliability of the system, delivery times, safety and equipment effectiveness are also needed to the companies of world class category and above mentioned benefits cannot be achieved by practising merely single maintenance system but their integration may do.

Lot of investigations have been carried out on individual maintenance system which determined the optimum cost of maintenance, threshold value where preventive maintenance must be carried out, optimum inspection schedule etc. [1-3] but very few literature is reported in the area of integration of various maintenance systems [4-6].

G. Niu et al. [4] has proposed a novel condition-based maintenance system that uses reliability-centered maintenance mechanism to optimize maintenance cost, and employs data fusion strategy for improving condition monitoring, health assessment, and prognostics. X. Zhou et al. [5] has proposed a reliability centered predictive maintenance policy for a system subject to degradation due to the imperfect maintenance effect and where a hybrid hazard rate recursion rule based on the concept of age reduction factor and hazard rate increase factor is assumed. In some papers, a reliability centered maintenance 
has been implemented by using vibration analysis as condition based monitoring technique[6].

In this paper, an integration of maintenance systems such as Condition Based Maintenance(CBM), Reliability Centered Maintenance (RCM) and Total Productive Maintenance (TPM) is proposed for a continuously monitored system subject to degradation due to the imperfect maintenance, where a hybrid hazard rate based on the concept of age reduction factor and hazard rate increase factor is used to predict the evolution of the system reliability in different maintenance cycles. A maintenance policy is suggested for an integrated maintenance system. A mathematical model for an integrated maintenance system is also derived in order to assess the performance of the proposed maintenance policy.

\section{Maintenance systems}

All the maintenance systems such as TPM,RCM and CBM [2,7,8,] are comprehensive and self-sufficient in their approach but their benefits are different from each other to some extent. To obtain the benefits such as minimum cost of maintenance, equipment effectiveness, reliability, quality, delivery time, flexibility, safety etc. at a time from these maintenance systems their integration may be explored.This can be done by inducting some features of a maintenance system into other or by fusing some features of different maintenance systems into one and then agumenting the same into proposed maintenance system.

Total productive maintenance is a companywide activity and, therefore, the focus is widespread. But integrated maintenance systems relies on the Maintenance Department and machine operators only. However, it does not mean that there is no involvement of higher management in integrated management systems, but definitely their role shifted from active participation to supportive participation.

In Integrated Maintenance System, first, few important features of TPM such as establishment of $5 \mathrm{~s}$, autonomous maintenance program and office TPM are required to implement, then RCM and finally CBM as shown in figure 1.

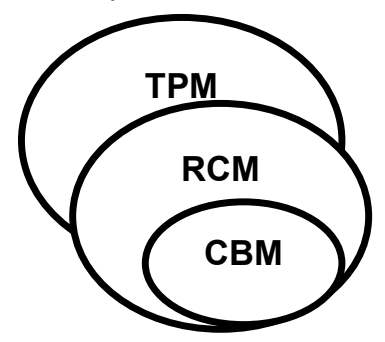

Figure1. Integration of CBM, RCM \& TPM

\section{Maintenance policy for an integrated maintenance system}

In Integrated Maintenance System, initially the TPM pillars such as establishment of 5s within the company, employ autonomous maintenance program are required to implement then the RCM system should be used. In RCM not only the critical component of the system from an operational point of view has to be determined and maintained, but the component of the system which is critical to Quality of product has also to be determined and maintain. Here, the omitted pillars of TPM such as Kaizen, planned maintenance, 
Training, Quality Maintenance and Safety Environment will be taken care of by maintenance personnel. Then the CBM is suggested and finally office TPM must be used to avoid administrative losses. The integrated maintenance policy is given below.

- Establish 5s within the company.

- Employ autonomous maintenance program.

- Establish boundaries.

- Identify interfaces and Specify important functions.

- Identify dominant and critical failure modes.

- Identify dominant failure causes.

- Select maintenance tasks (Use condition monitoring technique wherever possible).

- Predict the failure of a component and accordingly decide the next inspection date.

- Office TPM.

\section{Hybrid hazard rate and quantitative decision making model}

The following notations have been used, while formulating an effective maintenance model, using Hybrid hazard rate [9].

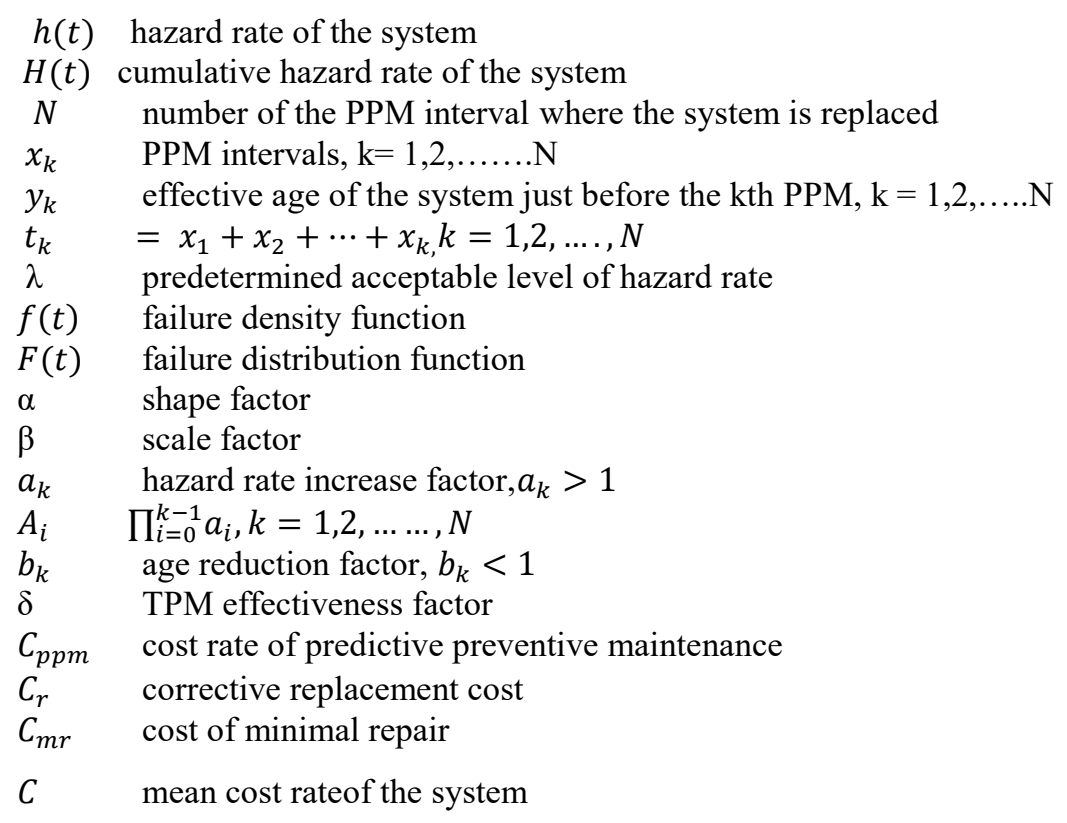

\subsection{Basic assumptions}

While constructing such a model for Hybrid hazard rate and degraded effectiveness of an asset, it is necessary to make some relevant assumptions, as summarized below:

i. The system is continuously and perfectly monitored and the monitoring has no effect on system's performance.

ii. The planning horizon is infinite.

iii. Failure rate of the system is an increasing function over time. It implies that the system deteriorates with time, as well as, with the number of PPM carried out. After each PPM, the system is operational but its failure characteristics are altered. 
iv. The system undergoes minimal repair at failures between maintenance cycles; the hazard rate remains unchanged by minimal repair.

v. The time for Predictive preventive maintenance,minimal repair and replacement are negligible.

vi. The system is restored to as good as new state at replacement.

\subsection{Model for an integrated maintenance system}

To know the effect of implementation of TPM a subjective scale ranging from 0.1 to 1 is used. 0.1 means very-very good effect of TPM implementation and 1 means very-very bad or no effect of TPM implementation. In this paper,the reliability of the system is converted into the hazard rate for the purpose of analysing the system. We consider the situation where a system is preventively maintained at $t_{1}, t_{2}, \ldots, t_{N-1}$ and replaced at $t_{N}$. Minimal repair is performed at failures between PPMs.Replacement of the system restores the system to as good as new.The system has the hazard rate $A_{k} h(t)$ between the $(k-1)$ thand the $k$ th PPMs i.e.,in time interval $\left(t_{k-1}, t_{k}\right)$. The effective age of the system becomes $b_{k-1} y_{k-1}$ right after the $(k-1)$ th PPM and then becomes $y_{k}=x_{k}+b_{k-1} x_{k-1}+\cdots+$ $b_{k-1} b_{k-2} \ldots b_{2} b_{1} x_{1}$ immediately before the $k$ th PPM:i.e., the effective age of the system changes from $b_{k-1} y_{k-1}$ to $y_{k}$ in $\left(t_{k-1}, t_{k}\right)$. Obviously we have $y_{k}=x_{k}+$ $b_{k-1} y_{k-1}$.Following Ref.[10] an integrated quantitative decision making model is given below.

$$
C=\frac{\left[c_{r}+c_{p p m}(N-1)+c_{m r} \sum_{k=1}^{N} \int_{b_{k-1} y_{k-1}}^{y_{k}} A_{k} h_{\left(x_{k}\right)} d x\right] \delta}{\left[\sum_{k=1}^{N-1}\left(1-b_{k}\right) y_{k}+y_{N}\right]}
$$

In this model,PPM is performed whenver the hazard rate of the system reaches the predetermined level $\lambda$ and the decision variable is $N$.This implies that the hazard rate at time $t_{i}(i=1,2, \ldots, N)$ must equal to $\lambda[11]$; that is,

$$
A_{k} h_{\left(y_{k}\right)}=\lambda, k=1,2, \ldots, N \text {. }
$$

Solving equation(4) with respect to $y_{k}(k=1,2, \ldots, N)$, we get the expressions of $\lambda$.Substituting these expressions into equation (3). Then $C$ becomes a function of $\lambda$. Differentiating $C$ with respect to $\lambda$ and setting it to zero leads

$$
\frac{\left[\sum_{k=1}^{N-1} \frac{h_{\left.\left(y_{k}\right)-a_{k} b_{k} h_{\left(b_{k}\right.} y_{k}\right)}^{h^{h}\left(y_{N}\right)}}{h^{\prime}\left(y_{k}\right)}+\frac{h^{\prime}\left(y_{N}\right)}{h^{\prime}}\right.}{\sum_{k=1}^{N-1} \frac{1-b_{k}}{A_{k} h^{\prime}\left(y_{k}\right)}+\frac{1}{A_{N} h^{\prime}\left(y_{N}\right)}}=\frac{C}{C_{m r}}
$$

where $y_{k}(k=1,2, \ldots, N)$ is a function of $\lambda$. Solving equation (5) with respect to $\lambda$,we obtain $\lambda$ as a function of N.Than we can find $\mathrm{N}$ which minimizes

$$
\frac{\left[\sum_{k=1}^{N-1} \frac{{ }^{h}\left(y_{k}\right)-{ }^{a_{k} b_{k}{ }^{h}\left(b_{k} y_{k}\right)}+\frac{{ }^{h}\left(y_{N}\right)}{h^{\prime}\left(y_{k}\right)}+\frac{h^{\prime}\left(y_{N}\right)}{h^{\prime}}}{\sum_{k=1}^{N-1} \frac{1-b_{k}}{A_{k} h^{\prime}\left(y_{k}\right)}+\frac{1}{A_{N} h^{\prime}\left(y_{N}\right)}}\right.}{\delta}
$$

where $y_{k}(k=1,2, \ldots, N)$ is a function of $\lambda$ and thus a function of $\mathrm{N}$ 


\subsection{Numerical example}

We consider the case where the hazard rate function of the system is Weibull,i.e.,

$$
h(t)=\beta t^{\alpha-1}, \alpha>1, \beta>0 .
$$

Let $\frac{C_{r}}{C_{p p m}}=2,5,10,20,50, \frac{C_{m r}}{C_{p p m}}=4, \alpha=2, \beta=3, \delta=0.7$ and $a_{k}=\frac{6 k+1}{5 k+1}, b_{k}=\frac{k}{2 k+1}, k=$ $0,1,2 \ldots$

Solving equation (4) with respect to $y_{k}(k=1,2, \ldots, N)$, we have

$$
y_{k}=\left(\frac{\lambda}{A_{k} \beta}\right)^{\frac{1}{\alpha-1}}, k=1,2,
$$

Substituting equation (7) into Eq.(5) and solving ti with respect to $\lambda$, we get

$$
\lambda=\beta^{\frac{1}{\alpha}}\left[\frac{C_{r}+C_{p p m}(N-1)}{\left(1-\frac{1}{\alpha \delta}\right) C_{m r} E(N) \delta}\right]^{\frac{\alpha-1}{\alpha}}
$$

where $E(N)=\sum_{k=1}^{N-1}\left(1-a_{k} b_{k}^{\alpha}\right) A_{k}^{-\frac{1}{\alpha-1}}+A_{N}^{-\frac{1}{\alpha-1}}$

Then expression (6) becomes

$$
\beta^{\frac{1}{\alpha}}\left[\frac{C_{r}+C_{p p m}(N-1)}{\left(1-\frac{1}{\alpha \delta}\right) C_{m r} \delta}\right]^{\frac{\alpha-1}{\alpha}} \frac{[E(N)]^{\frac{1}{\alpha}}}{F(N)}
$$

where $F(N)=\sum_{k=1}^{N-1}\left(1-b_{k}\right) A_{k}^{-\frac{1}{\alpha-1}}+A_{N}^{-\frac{1}{\alpha-1}}$

Minimization of the function in expression (9)is equivalent to minimization of function

$$
Q(N)={\frac{\left[C_{r}+C_{p p m}(N-1)\right]^{\frac{\alpha-1}{\alpha}}}{F(N)}}^{[}[E(N)]^{\frac{1}{\alpha}}
$$

Inequalities $Q(N+1) \geq Q(N)$ and $Q(N)<Q(N-1)$ imply that

$$
W(N) \geq \frac{C_{r}}{C_{p p m}} \text { and } W(N-1)<\frac{C_{r}}{C_{p p m}}
$$

where

$$
W(N)=\frac{[E(N+1)]^{\frac{1}{\alpha-1}}[F(N)]^{\frac{\alpha}{\alpha-1}}}{[E(N)]^{\frac{1}{\alpha-1}}[F(N+1)]^{\frac{\alpha}{\alpha-1}}[E(N+1)]^{\frac{1}{\alpha-1}}[F(N)]^{\frac{\alpha}{\alpha-1}}}-(N-1)
$$

Thus, the optimal value $\mathrm{N}^{*}$ must satisfy inequalities(10). Table1 shows the optimal PPM schedules, $\mathrm{N}^{*}, x_{1}, x_{2}, x_{3}, \ldots x_{N *}$. Theoptimal PM intervals are computed from $x_{k}=y_{k}-$ $b_{k-1} y_{k-1}(k=1,2, \ldots, N)$.

Table 1. Optimal PPM schedules for integrated maintenance systems

\begin{tabular}{|c|c|c|c|c|c|}
\hline $\boldsymbol{C}_{r} / \boldsymbol{C}_{p p m}$ & $\mathbf{2}$ & $\mathbf{5}$ & $\mathbf{1 0}$ & $\mathbf{2 0}$ & $\mathbf{5 0}$ \\
\hline $\mathbf{N}^{*}$ & $\mathbf{1}$ & $\mathbf{3}$ & $\mathbf{5}$ & $\mathbf{8}$ & 11 \\
\hline
\end{tabular}




\begin{tabular}{|c|c|c|c|c|c|}
\hline$x_{1}$ & .67 & 1.12 & 1.46 & 1.7 & 2.40 \\
$x_{2}$ & & .59 & .67 & .89 & 1.92 \\
$x_{3}$ & & .43 & .51 & .65 & .92 \\
$x_{4}$ & & & .33 & .50 & .72 \\
$x_{5}$ & & .32 & .41 & .58 \\
$x_{6}$ & & & .33 & .47 \\
$x_{7}$ & & & .27 & .38 \\
$x_{8}$ & & & .22 & .32 \\
$x_{9}$ & & & & .26 \\
$x_{10}$ & & & & .22 \\
$x_{11}$ & & & & \\
\hline
\end{tabular}

\section{Conclusion}

Excellence in maintenance can be achieved by integrating various maintenance systems such as CBM, RCM and TPM, which in-turn gives minimum maintenance cost, flexibility, quality, delivery time, equipment effectiveness, reliability, safety etc. and the effectiveness of integrating the maintenance systems can be evaluated by quantitative decision making model. From Table1,we see that $x_{k}$ decreases for all $k$ values. Because of the introduction of TPM effectiveness factor $\delta$ the PPM time intervals of our model are greater than the time intervals mentioned in Ref.

$[10,11]$ but it also suggests to do more frequent PPM maintenance with age as suggested in above references.In our model we considered hybrid hazard rate which actually expedite the process of deterioration of the system but then also the hazard rate takes more time to reach the threshold value because of the introduction of TPM system into maintenance activity.The hazard rate at every PPM remains the same (equals $\lambda$ ). That is, $\lambda$ is set to be a specific level.Then $y_{k}(k=1,2, \ldots, N)$ can be computed from Eq.(2). The mean cost rate $\mathrm{C}$ is a function only of $\mathrm{N}$. Thus, we can find $\mathrm{N}$ which minimizes $\mathrm{C}$ and finally obtain the optimal PPM schedule.

The proposed maintenance model can be implemented by taking a real time case and a mathematical model can be used for checking the improvement in condition as a future scope.

\section{References}

[1] A. Grall, M. Fouladirad, Risk \& Reliab., 222(special issue), 359-369 (2008)

[2] A. Grall, C. Berenguer, L. Dieulle, Reliab. Engg. Syst. Saf., 76,167-180,( 2002)

[3] Venkatesh J., Plant Maintenance Resource Center, (1996-2005)

[4] G. Niu, B S Yang, M. Pecht, Reliab. Engg. Syst. Saf., vol.95, 786-796 (2010)

[5] X. Zhou, L. Xi , J. Lee, Reliab. Engg. Syst Saf., vol.92, 530-534(2007)

[6] S. Parida, N.R. Kotu, M. Prasad, Roma $\mathbf{1 5}^{\text {th }}$ WCNDT(2000)

[7] S. Nakajima, Productivity Press (India) private ltd., first Indian edition, (1993)

[8] I.H. Afefy, Reliab. Engg.Syst. Saf., vol.60, No.2, 863-873(1998)

[9] RV Canfield, IEEE Trans Reliab., 35(1), 78-8 (1986)

[10] T. Nakagawa, IEEE Transactions on Reliab., R-37, 295-298(1988)

[11] D. Lin, M.J. Zuo, R.C.M. Yam, Int., journal of Reliab. Qua. Saf. Engg.,vol.7,No.3, 253-266 (2000) 